\title{
Fractional Order Control for Aeration of Activated Sludge Wastewater Treatment Processes
}

\author{
Li Meng ${ }^{1, a}$, Xuelian Zhang ${ }^{2, b}$, Ashu Wei ${ }^{2, ~ c}$, and Xiaowei Han ${ }^{1, d}$ \\ ${ }^{1}$ Department of Information Engineering, Shenyang University, Shenyang, 110044, China \\ ${ }^{2}$ Shenshuiwan Sewage Treatment Plant of Guodian Northeast Environmental Protection Industry \\ Group Co., Ltd., Shenyang, 110141, China \\ a mengliinsy@126.com, b105438758@qq.com, ${ }^{\mathrm{c}} 391077784 @ q q . c o m,{ }^{d} \mathrm{hxw69@163.com}$
}

Keywords: Fractional Order; Activated Sludge Process; dissolved oxygen concentration

\begin{abstract}
In this paper, the design scheme of fractional order proportional integral (FOPI) controller for the second order plus time delay model of aeration of activated sludge wastewater treatment process are presented. It is found that the two controllers designed by the proposed tuning method not only make the system stable, but also improve the performance for the aeration control of activated sludge wastewater treatment processes. Simulation results are presented to validate the proposed tuning schemes. Furthermore, from the simulation results, it can be seen that the fractional order PI controller outperforms the integer order PID controller.
\end{abstract}

\section{Introduction}

Activated Sludge Process (ASP) is one of the widely used sewage treatment methods. It consists of two parts: reaction cell (aeration tank) and sedimentation tank (shown in Fig. 1).

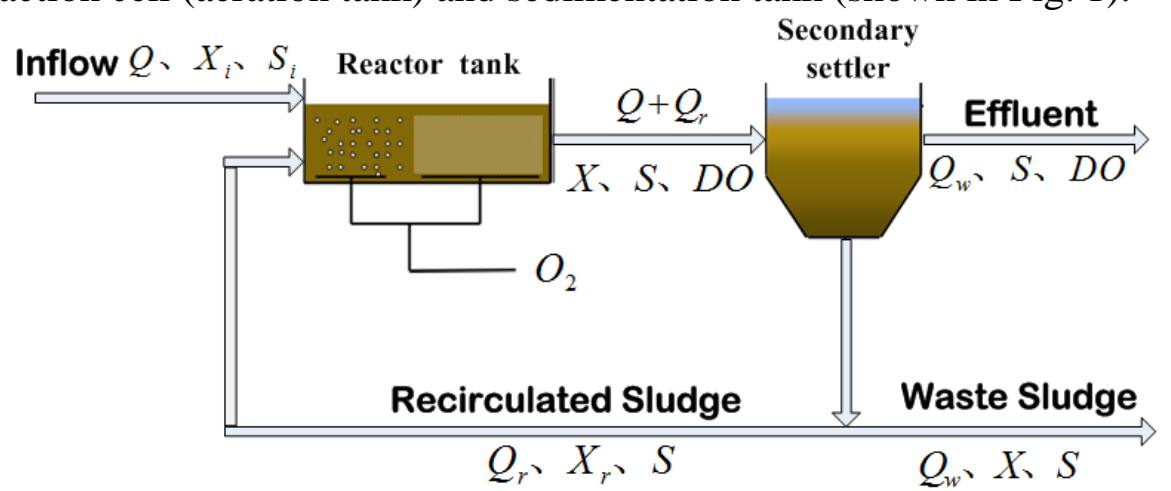

Fig.1. Simple configuration for the activated sludge process

The activated sludge process uses microorganisms to feed on organic contaminants in wastewater, producing a high-quality effluent [1]. The basic principle behind all activated sludge processes is that as microorganisms grow, they form particles that clump together. These particles (floc) are allowed to settle to the bottom of the tank, leaving a relatively clear liquid free of organic material and suspended solids. Described simply, screened wastewater is mixed with varying amounts of recycled liquid containing a high proportion of organisms taken from a secondary clarifying tank, and it becomes a product called mixed liquor. This mixture is stirred and injected with large quantities of air, to provide oxygen and keep solids in suspension. After a period of time, mixed liquor flows to a clarifier where it is allowed to settle. A portion of the bacteria is removed as it settles, and the partially cleaned water flows on for further treatment. The resulting settled solids, the activated sludge, are returned to the first tank to begin the process again.

Aeration is the most critical component of a treatment system using the activated sludge process. A well designed aeration system has a direct impact on the level of wastewater treatment it achieves. An ample and evenly distributed oxygen supply in an aeration system is the key to rapid, economically-viable, and effective wastewater treatment. Maintaining the dissolved oxygen 
concentration (DO) at a certain setpoint represents one of the major concerns of researchers in ASP control[2]. This is due to the fact that a very low dissolved oxygen concentration in the aeration tank results in biomass die-off, while an excessively high dissolved oxygen level increases energy consumption and may degrade the sludge quality. Operators typically control the aeration rate to assure a concentration of $2-3 \mathrm{mg} / \mathrm{L}$ of dissolved oxygen (D.O.) at the discharge end of the aeration tank.

Many research papers have been written with the goal of improving the performance of real, existing ASP from different countries by implementing controllers for the DO concentration [3-5].Other researchers have emphasized the importance of well tuned models and simulation platforms in the process of designing controllers for the plant $[5,6]$, while some have implemented multiple model controllers instead of complex nonlinear ones [7].

For decades the PID controller has been the most common control algorithm because of its obvious advantages: having a simple clear structure and being suitable for most control loops. For ASP, a simple PID controller might prove to be insufficient in maintaining a desired setpoint, considering the changes in the ASP operating point. Several more robust and complex strategies have been designed to tackle this issue, such as adaptive [8], predictive [9] or fuzzy [10] control.

An alternative approach to the control of the dissolved oxygen concentration is proposed in this paper, and it is based on the fractional calculus. This approach has received considerable attention during the past decade in engineering applications, including both process modeling $[11,12]$ and controller design [13-15]. The fractional order $P I^{\lambda} D^{\mu}$ controller is in fact a generalization of the integer order PID controller, having the order of integration $\lambda$ and the order of differentiation $\mu$. The main interest regarding fractional order controllers is due to the supplementary tuning parameters involved, $\lambda$ and ${ }^{\mu}$, which can be used to increase the overall robustness of the closed loop system [14-16].

In this paper, fractional order PI controller is designed for the aeration control of activated sludge process.

\section{Process Model of the sewage treatment process of activated sludge}

As a biochemical reaction process, dissolved oxygen is a very important parameter can reflect, intuitively and quickly, the operating conditions of the entire sewage treatment system. The IAWQ (International Association on Water Quality) has established ASM1 model for the removal of carbon and nitrogen from sewage sludge. The correlation rate in the main biological processes, such as carbon oxidation, nitrification and denitrification in Sequencing Batch Reactor Activated Sludge Process (SBR), is quantitatively described. The following dissolved oxygen transfer model is established [17]

$$
V \frac{d c}{d t}=Q C_{0}-Q C_{1}-V K C
$$

where $\mathrm{V}$ is the volume of the reaction cell. $\mathrm{Q}$ is the air flow rate. $\mathrm{K}$ is the reaction rate constant. $\mathrm{C}$ is the dissolved oxygen concentration in the reaction cell. $\mathrm{C} 0$ is the dissolved oxygen concentration of the air blown into the air. C1 is the dissolved oxygen concentration in the exhaust gas.

In actual sewage treatment process, the dissolved oxygen is adjusted by the aeration control with the frequency converter and the air blower. Combined with the time-delay of detection and control response of DO, the mathematical model [18] is established

$$
G(s)=\frac{k}{a_{1} s^{2}+a_{2} s+1} e^{-L s}
$$

where $k=1, a_{1}=120000, a_{2}=1300, L=30$.

According to the system mathematical model (1), we can get its frequency response,

$$
P(j \omega)=\frac{k}{\left(1-a_{1} \omega^{2}\right)+j a_{2} \omega} e^{-j \omega L}
$$




\section{Structure and Design Specifications of Fractional Order Controllers}

Assume that the gain crossover frequency is given by $\omega_{c}$ and phase margin is specified by $\phi_{m}$. To ensure the system stability and robustness, three specifications are proposed as follows [14],

(1) Phase margin specification

At the gain crossover frequency of $\omega_{c}$, one has

$$
\begin{aligned}
\left|G\left(j \omega_{c}\right)\right| & =\left|C\left(j \omega_{c}\right) P\left(j \omega_{c}\right)\right|=1 \\
\arg \left[G\left(j \omega_{c}\right)\right] & =\arg \left[C\left(j \omega_{c}\right) P\left(j \omega_{c}\right)\right]=-\pi+\phi_{m}
\end{aligned}
$$

where $\phi_{m}$ is referred as the phase margin of the system, $\arg (\cdot)$ is the argument.

(2) Robustness variations in the plant gain

The constraint is discussed in

$$
\left(\frac{d(\arg [G(j \omega)])}{d \omega}\right)_{\omega=\omega_{c}}=\left(\frac{d(\arg [C(j \omega) P(j \omega)])}{d \omega}\right)_{\omega=\omega_{c}}=0
$$

This constraint forces the gain around $\omega_{c}$ to go flat, such that the phase remains within a neighborhood of the specified phase margin, such that for the variations in the plant gain, the closed-loop system reserves iso-damping behavior.

In this study, we consider three simple controllers: the integer order PID control and fractional order PI controllers whose transfer functions are respectively given in the following:

$$
\begin{array}{r}
C_{1}(s)=K_{p}+\frac{K_{i}}{s}+K_{d} s \\
C_{2}(s)=K_{p}\left(1+\frac{K_{i}}{s^{\lambda}}\right)
\end{array}
$$

\section{Designs of Integer Order PID and Fractional Order PI Controller for the control of dissolved oxygen concentration}

1) IOPID Controller Design

According to the integer order PID controller transfer function (6), we can get its frequency response as follows,

$$
C_{1}(j \omega)=K_{p}+j\left(K_{d} \omega-\frac{K_{i}}{\omega}\right)
$$

The phase and gain are as follows,

$$
\arg \left[C_{1}(j \omega)\right]=\arctan \left(\frac{K_{d} \omega^{2}-K_{i}}{K_{p} \omega}\right) \quad\left|C_{1}(j \omega)\right|=\sqrt{K_{p}^{2}+\left(\frac{K_{d} \omega^{2}-K_{i}}{\omega}\right)^{2}}
$$

The phase derivative of the IOPID

$$
\frac{d\left(\arg \left[C_{1}(j \omega)\right]\right)}{d \omega}=\frac{d}{d \omega}\left[\arctan \left(\frac{K_{d} \omega^{2}-K_{i}}{K_{p} \omega}\right)\right]=\frac{1}{1+\left(\frac{K_{d} \omega^{2}-K_{i}}{K_{p} \omega}\right)^{2}} \cdot \frac{K_{d} \omega^{2}+K_{i}}{K_{p} \omega^{2}}
$$

According to specifications (3)-(5), we can gain

$$
\begin{gathered}
\frac{K_{d} \omega_{c}^{2}-K_{i}}{K_{p} \omega_{c}}=A_{1} \\
\frac{1}{1+A_{1}^{2}} \cdot \frac{K_{d} \omega_{c}^{2}+K_{i}}{K_{p} \omega_{c}^{2}}=B_{1} \\
K_{p}=\frac{C_{1}}{\sqrt{1+A_{1}^{2}}}
\end{gathered}
$$


where $A_{1}=\tan \left(\phi_{m}-\arg \left[P\left(j \omega_{c}\right)\right]\right)$ is a constant. $B_{1}=-(d(\arg [P(j \omega)]) / d \omega)_{\omega=\omega_{c}}$ and $C_{1}=1 /\left|P\left(j \omega_{c}\right)\right|$. From (9), (10) and (11), we can get,

If the parameters are set as follows,

$$
\begin{gathered}
K_{d}=\frac{C_{1}}{2 \sqrt{1+A_{1}^{2}}}\left[B_{1}\left(1+A_{1}^{2}\right)+\frac{A_{1}}{\omega_{c}}\right] \\
K_{i}=\frac{C_{1} \omega_{c}^{2}}{2 \sqrt{1+A_{1}^{2}}}\left[B_{1}\left(1+A_{1}^{2}\right)-\frac{A_{1}}{\omega_{c}}\right]
\end{gathered}
$$

then we can get directly from (11)-(13)

$$
\omega_{c}=0.01 \mathrm{rad} / \mathrm{s}, \phi_{m}=60^{\circ}
$$

$$
K_{d}=1239, K_{i}=0.04546, K_{p}=15.1155 .
$$

The IOPID controller is

$$
C_{1}(s)=15.1155+\frac{0.04546}{s}+1239 s
$$

We can fix the open-loop frequency response with the integer order PID controller. So, the Bode diagrams of system designed can be plotted as in Fig. 2, we can see that the phase Bode plot is flat, at the gain crossover frequency. All the three specifications are satisfied.

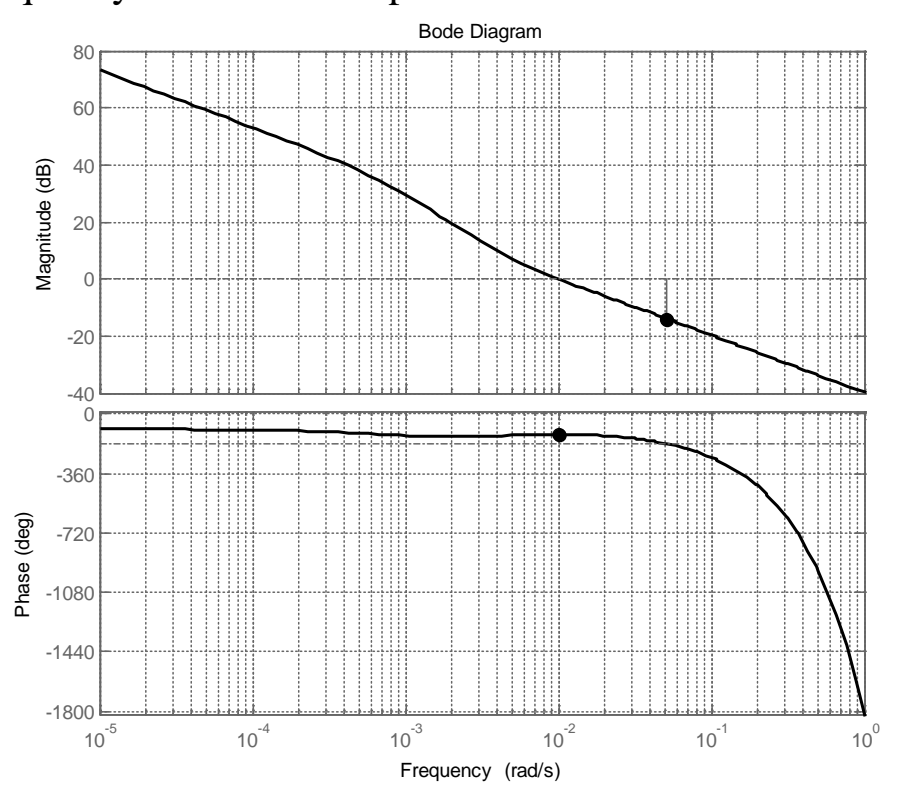

Fig. 2. Open-loop Bode diagram with the IOPID controller

2) FOPI Controller Design

According to the fractional order PI controller transfer function (7), we can get its frequency response as follows,

$$
C_{2}(j \omega)=K_{p}\left(1+K_{i}(j \omega)^{-\lambda}\right)=K_{p}\left(1+K_{i} \omega^{-\lambda} \cos \left(\lambda \frac{\pi}{2}\right)-j K_{i} \omega^{-\lambda} \sin \left(\lambda \frac{\pi}{2}\right)\right)
$$

The phase and gain are as follows,

$$
\arg \left[C_{2}(j \omega)\right]=-\arctan \left(\frac{K_{i} \omega^{-\lambda} \sin \left(\lambda \frac{\pi}{2}\right)}{1+K_{i} \omega^{-\lambda} \cos \left(\lambda \frac{\pi}{2}\right)}\right) \quad\left|C_{2}(j \omega)\right|=K_{p} \sqrt{1+2 K_{i} \omega^{-\lambda} \cos \left(\lambda \frac{\pi}{2}\right)+K_{i}^{2} \omega^{-2 \lambda}}
$$

The phase derivative of the FOPI 


$$
\frac{d\left(\operatorname{Arg}\left[C_{2}(j \omega)\right]\right)}{d \omega}=\frac{d}{d \omega}\left[-\arctan \left(\frac{K_{i} \omega^{-\lambda} \sin \left(\lambda \frac{\pi}{2}\right)}{1+K_{i} \omega^{-\lambda} \cos \left(\lambda \frac{\pi}{2}\right)}\right)\right]=\frac{1}{1+\left(\frac{K_{i} \omega^{-\lambda} \sin \left(\lambda \frac{\pi}{2}\right)}{1+K_{i} \omega^{-\lambda} \cos \left(\lambda \frac{\pi}{2}\right)}\right)^{2}} \cdot \frac{\lambda K_{i} \omega^{-\lambda-1} \sin \left(\lambda \frac{\pi}{2}\right)}{\left(1+K_{i} \omega^{-\lambda} \cos \left(\lambda \frac{\pi}{2}\right)\right)^{2}}
$$

According to specifications (3)-(5), we can gain

$$
\begin{gathered}
K_{i}=\frac{-A_{1} \omega_{c}^{\lambda}}{\left[\sin \left(\lambda \frac{\pi}{2}\right)+A_{1} \cos \left(\lambda \frac{\pi}{2}\right)\right]} \\
K_{i}=\frac{\lambda A_{1}^{2} \omega_{c}^{\lambda-1}}{B_{1}\left(1+A_{1}^{2}\right) \sin \left(\lambda \frac{\pi}{2}\right)} \\
K_{p}=\frac{C_{1}}{\sqrt{1+2 K_{i} \omega_{c}^{-\lambda} \cos \left(\lambda \frac{\pi}{2}\right)+K_{i}^{2} \omega_{c}^{-2 \lambda}}}
\end{gathered}
$$

Plot curve 1, $K_{i}$ w.r.t $\lambda$ according to (16), and plot curve 2, $K_{i}$ w.r.t $\lambda$ according to (17) are both shown in Fig. 3(a). $\lambda$ and $K_{i}$ are obtained from the intersection point on the above two curves, which reads $\lambda=1.8504, K_{i}=3.8034 \times 10^{-4}$. Then $K_{p}=9.2957$ is calculated from (18).

$$
C_{2}(s)=9.2957\left(1+\frac{3.8034 \times 10^{-4}}{s^{1.8504}}\right)
$$

The Bode plots of open-loop control system designed are shown in Fig. 3(b), where we can see that the phase Bode plot is flat at the gain crossover frequency and all three specifications are satisfied precisely.

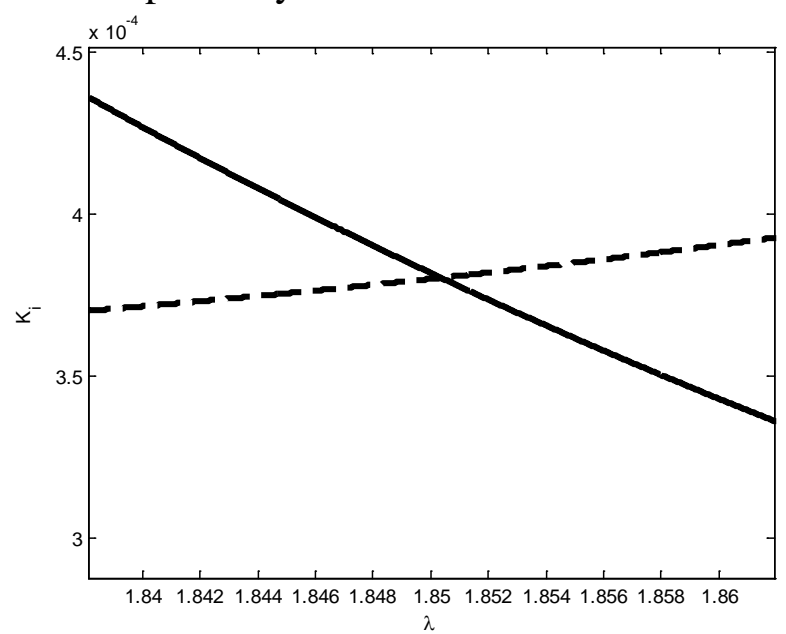

(a)
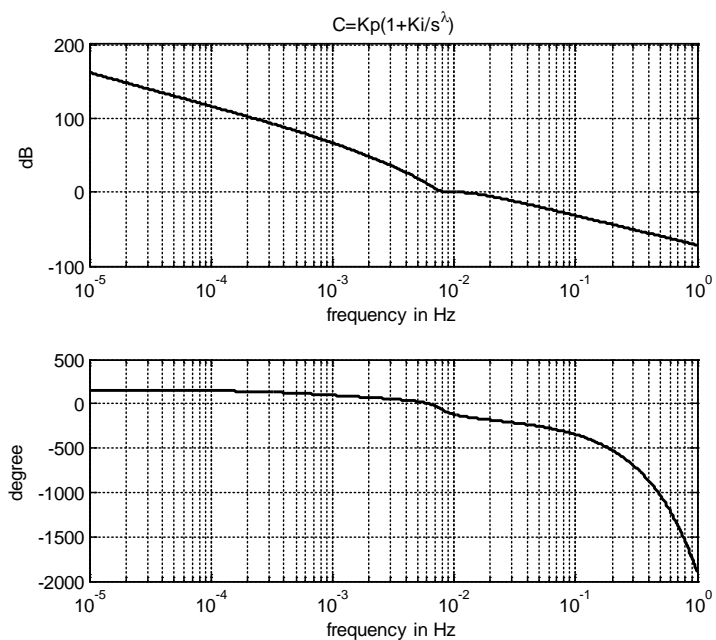

(b)

Fig.3. Open-loop Bode diagram with the FOPI controller

\section{Simulation}

In this section, the tuning methods presented above are validated via the numerical simulation. The step response of the fractional order system is implemented by the inverse laplace function INVLAP.m.

As shown in Fig. 4 and Fig. 5, using the IOPID controller and FOPI controller, the closed-loop step responses are plotted with various open-loop gains $k$.

It can be seen from Fig. 4 and Fig. 5 that the integer order PID and the fractional order PI 
controllers designed by the proposed method in this paper are effective.

The step responses remain stable under gain variations, which mean the system is robust to gain changes. From Fig. 4 and Fig.5, we can see that the settling time of the unit step response using the FOPI controller is even much shorter than those using the IOPID controllers with the designed parameters. Thus, we can conclude that the FOPI controller outperforms the IOPID controllers.

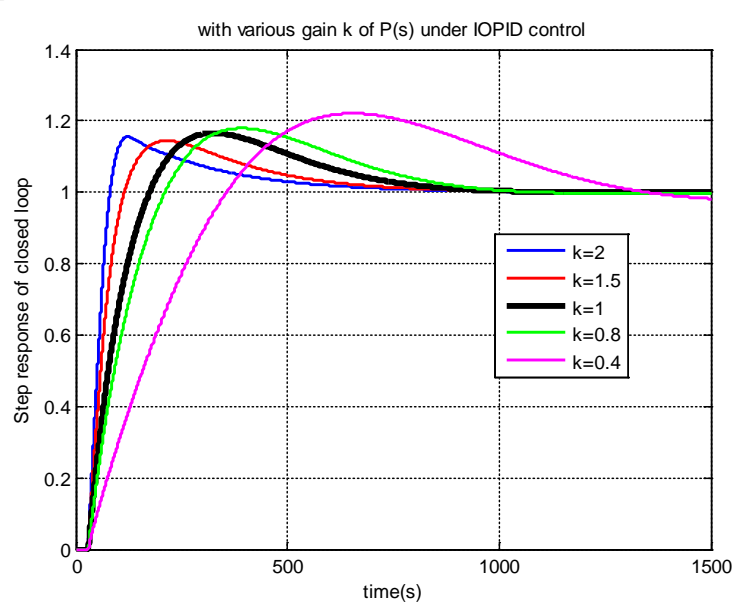

Fig.4. Step response of the IOPID controlled system with various gains

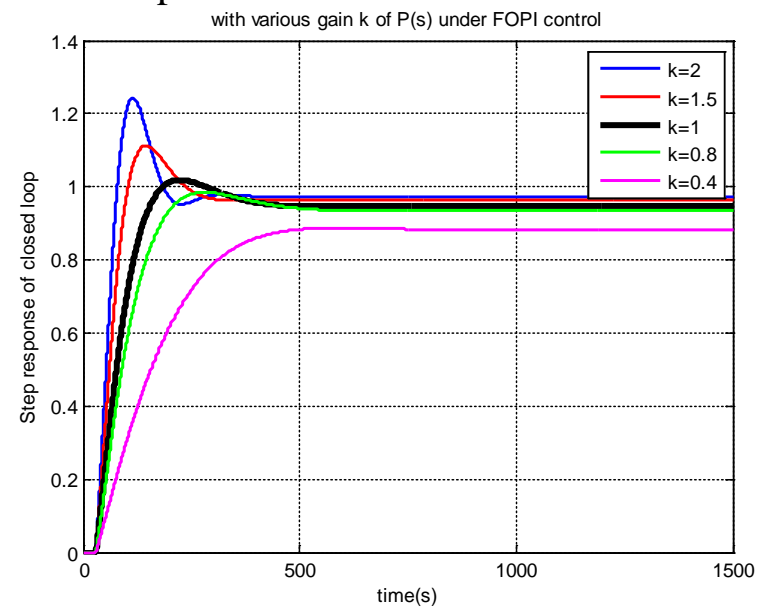

Fig.5. Step response of the FOPI controlled system with various gains

\section{Conclusion}

In this paper, we presented the design schemes of IOPID controller and FOPI controller for aeration control of activated sludge wastewater treatment process. The controller tuning method ensure that the given gain crossover frequency and the phase margin are achieved, and the phase derivative w.r.t. the gain crossover frequency is zero, i.e., the phase Bode plot is flat at the gain crossover frequency. The two controllers designed by the proposed tuning method not only make the system stable, but also improve the performance and robustness for the second order plus delay time system. Simulation results are presented to validate the proposed tuning schemes. Furthermore, from the simulation results, it can be seen that the FOPI controller outperforms the the integer order PID controller.

\section{Acknowledgements}

This work was financially supported by Liaoning Building Project of Public Welfare Research Team in Science (GY2015-E-009) and Shenyang Program for Tackling Key Problems in Science and Technology (F14-129-9-00).

\section{References}

[1]. D. Brjdanovic, S.C.F.Meijer, C.M. Lopez-Vazquez, C.M. Hooijmans, M.C.M. van Loosdrecht, Applications of Activated Sludge Models (IWA Publishing, London, 2015)

[2]E. Pittoors, Y. Guo, S.W.H. Van Hulle, Modeling dissolved oxygen concentration for optimizing aeration systems and reducing oxygen consumption in activated sludge processes: a review. Chem. Eng. Commun. 201(8), 983-1002 (2014)

[3]. G. Harja , G. Vlad, I. Nascu, Dissolved oxygen control strategy for an activated sludge wastewater treatment process. Recent Advances in Electrical Engineering Series. Recent Advances in Systems, in Proceedings of the 19th International Conference on Systems (CSCC'15), 16-20 July, Zakynthos, Greece, pp. 453-458, ISSN:17905117, ISBN:978-1-61804-321-4, (2015)

[4]. M. Henderson, Energy Reduction Methods in the Aeration Process at Perth Waste Water Treatment Plant.University of Strathclyde, renewable energy systems and the environmentMSc 
individual theses, (2001-2002)

[5]. St Iordache, N. Petrescu, C. Necula, G. Busuioc, MunicipalWastewater treatment improvement using computer simulating, advances in waste management, in Proceedings of the 4th WSEAS International Conference on Waste Management, Water Pollution, Air Pollution, Indoor Climate (WWAI'10), pp. 95-100, ISBN: 978-960-474-190-8, (2010)

[6]. S. Kestel, T. Stahl, M. Gray, Process simulator evaluates blower and valve control strategies for WWTP aeration. Compressed air best practices. http://www. airbestpractices. com/industries / wastewater/ process-simulator-evaluates-blower-and-valve-control-strategies-wwtp-aeration, (2014)

[7]. J. Kocijan, N. Hvala, S. Strm cnik,Multi-model control of wastewater treatment reactor, in System and Control: Theory and Applications, ed. by N. Mastorakis. Electrical and Computer Engineering Series (World Scientific and Engineering Society, 2000), pp. 49-54

[8]. S. Mirghasemi, C.J.B.Macnab,A. Chu, Dissolved oxygen control of activated sludge bioreactors using neural-adaptive control, in IEEE Symposium onComputational Intelligence in Control and Automation, Orlando, Florida, pp. 1-6, (2014)

[9]. B. Holenda, E. Domokos, A. Redey, J. Fazakas, Dissolved oxygen control of the activated sludge wastewater treatment process using model predictive control. Comput. Chem. Eng. 32, 1270-1278 (2008)

[10] C. Belchior, R. Araújo, J. Landeck, Dissolved oxygen control of the activated sludge wastewater

treatment process using stable adaptive fuzzy control. Comput. Chem. Eng. 37, 152-162 (2011)

[11]. C. Ionescu, R. De Keyser, Relations between fractional order model parameters and lung pathology in chronic obstructive pulmonary disease. IEEE Trans. Biomed. Eng. 56, 978-987 (2009)

[12]. F. Mainardi, Fractional Calculus AndWaves in Linear Viscoelasticity: An Introduction toMathematical Models (Imperial College Press, London, 2010)

[13]. Y. Luo, Y.Q. Chen, C.Y. Wang, Y.G. Pi, Tuning fractional order proportional integral controllers for fractional order systems. J. Process Control 20, 823-831 (2010)

[14]. C.A. Monje, Y.Q. Chen, B.M. Vinagre, D. Xue, V. Feliu, Fractional order Systems and Controls: Fundamentals and Applications (Springer, London, 2010)

[15]. C.I. Muresan, S. Folea, G. Mois, E.H. Dulf, Development and implementation of an FPGA based fractional order controller for a DC motor. Elsevier J. Mechatron. 23(7), 798-804 (2013)

[16]. C. Copot, C.I Muresan, C.M. Ionescu, R. De Keyser, Fractional order control of a DC motor with load changes, in 14th International Conference on Optimization of Electrical and Electronic Equipment OPTIM, pp. 956-961, 22-24 May, Brasov, Romania, DOI:10.1109/OPTIM.2014.6850964 (2014)

[17]Jiang Yunwei, Wang Xiaoyi, Wei Wei, Xu Jiping and Wang Fan, Research of sewage treatment control method based on anti-disturbance and fuzzy PID[J]. Computers and Applied Chemistry, 2013,30(7):748-752.

[18]Wang Xiaoyi, Li Wandong and Liu Zaiwen. A control method of dissolved oxygen in sewage treatment based on fuzzy PID-smith[J]. Computer and Applied Chemistry, 2010, 27(6):801-803. 